\title{
EVALUATION OF INTRA-ARTICULAR INJECTION OF SIMVASTATIN VERSUS HYALURONIC ACID IN TREATMENT OF INTERNAL DERANGEMENT OF TEMPOROMANDIBULAR JOINT
}

\author{
Mohamed Ashraf Abdel Fattah Mahmoud ${ }^{1}$, Abdel Badee Abdallah Abdel Mabood ${ }^{2}$, \\ Gihan Gamal El Din El Desouky ${ }^{3}$, Eman Abdelhalim El Sharrawy ${ }^{4}$
}

DOI: $10.21608 / d s u .2021 .31287 .1037$

Manuscript ID: DSU-2005-1037

\section{KEYWORDS}

Arthrocentesis, internal derangement, simvastatin, sodium hyaluronate, TMJ.

- E-mail address: dr_m_ashraf87@yahoo.com

1. Postgraduate student in Oral and Maxillofacial Surgery Department, Faculty of Dentistry, Suez Canal University

2. Assistant Professor of Oral and Maxillofacial Surgery, Faculty of Dentistry, Zagazig University

3. Professor of Oral Radiology, Faculty of Dentistry, Suez Canal University

4. Professor of Oral and Maxillofacial Surgery, Faculty of Dentistry, Suez Canal University

\begin{abstract}
Introduction: TMJ Internal Derangement is a common form of temporomandibular joint disorder. Almost $80 \%$ of adult symptomatic patients with TMD have some form of ID. Temporomandibular joint arthrocentesis consists of lavage of upper joint space of TMJ done with no direct vision, aiming primarily to remove necrotic tissue, blood and pain mediators from the joint. One of these therapeutic substances is hyaluronic acid; which is a polysaccharide of the family of glycosaminoglycans, which can be found in many extracellular tissues, including synovial fluid and cartilage. Simvastatins are group of drugs which have good results in regeneration of degenerated TMJ. Aim: This study aimed to evaluate the intra-articular injection of simvastatin versus sodium hyaluronate following arthrocentesis for management of internal derangement (ID) of the temporomandibular joint(TMJ). Patients and methods: Thirty patients with internal TMJ derangement were included in this study. All patients were subjected to preoperative clinical examination and preoperative magnetic resonance imaging (MRI) for the affected joints. Arthrocentesis was performed firstly for the affected joints in all patients followed by intra-articular injection of $1 \mathrm{ml}$. simvastatin twice a month (in group I) and sodium hyaluronate twice a month ( in group II).Clinical follow up were done at 24 hours post-operatively, one week, one month, and six months. Then, after six months MRI was repeated to compare with preoperative images. Results: Better results were recorded with simvastatin group in comparison to sodium hyaluronate group; at 1 week, 1 month,and six months intervals; which was superior to arthrocentesis followed by sodium hyaluronate intra-articular injection. Patients in group I showed better MRI findings in comparison to group II where the position of the disc was almost returned to its normal anatomical position. Conclusion: Arthrocentesis followed by simvastatin intra-articular injection was superior to the combination of arthrocentesis and sodium hyaluronate intra-articular injection for management of TMJ internal derangements symptoms.
\end{abstract}

\section{INTRODUCTION}

TMJ is a complex joint that allows a range of movement of the associated structures. The free motion of an articular joint is essential for full function of the structures attached to that joint. Restriction of free movement of the joint can be due to pathology within the joint cavity, changes involving the capsule, disc, and also the activating muscles of the joint ${ }^{(1)}$. 
ID is a common form of TMJ disorder $\left(\mathrm{TMD}^{(2)}\right.$. The incidence of TMJ ID is very high in women between the second and fifth decade of life (18-45 years). Women in this age group also have a high incidence of TMJ clicking and tenderness. The female to male ratio in the population is between $3: 1$ to $10: 1$, with a high predisposition for women of reproductive age. Data from related literature has suggested that arthrocentesis may be of some benefit to manage the symptoms of TMDs. Such a technique was first introduced for the management of the sudden onset of closed lock ${ }^{(3,4)}$.

TMJ arthrocentesis consists of lavage of the upper joint space of TMJ done with no direct vision, aiming primarily to remove necrotic tissue, blood and pain mediators from the joint ${ }^{(5)}$. Arthrocentesis is a single most important non-invasive procedure in musculoskeletal medicine. It is abasic underlying procedure for intra-articular treatment, including therapeutic arthrocentesis, andintra-articular injection of therapeutic substances ${ }^{(6)}$.

One of these therapeutic substances is hyaluronic acid; which is a polysaccharide of the family of glycosaminoglycans, which can be found in synovial fluid. It is produced by chondrocytes and synoviocytes of TMJ, and in patients with osteoarthritis it becomes depolymerized, resulting in a decreased molecular weight and viscoelasticity. These modifications increase cartilage's susceptibility to injuries. Intra-articular injection of hyaluronic acid can stimulate the synthesis of endogenous hyaluronic acid, so reducing joint friction coefficient and decreasing the risk of damage ${ }^{(7)}$.

Simvastatins are group of drugs which have good results in regeneration of degenerated TMJ. Recently, it has been suggested for intraarticular injection of temporomandibular joint with promising results ${ }^{(8)}$. Accordingly, the present study hypothesized that the intraarticular injection of simvastatin was effective as hyaluronic acid injection in the treatment of patients with TMJ Internal Derangement.

\section{PATIENTS AND METHODS}

The current study was carried out on 30 adult patients suffering from internal derangement as approved by clinical and MRI examination. These patients were selected from those attending the outpatient clinic, of Oral and Maxillofacial surgery department of Faculty of Dentistry,Suez Canal University through the period from 2018 to 2019.

\section{Inclusion criteria:}

a. Adult patients over 18 years of age.

b. Patients diagnosed with internal derangement of TMJ.

c. Patients with a chief complaint of TMJ pain and limited maximum mouth opening.

d. Patient with internal derangement as revealed in their MRI examination.

e. Patients unresponsive to conservative treatment modalities for TMJ dysfunction .

\section{Exclusion criteria:}

a. Patients with a history of previous TMJ surgery.

b. Patients with limited mouth opening caused only by muscle spasm.

c. Patients with systemic inflammatory joint disease.

d. Patients with other pathological lesions in the joint.

e. Patients with direct trauma to the facial bones.

f. Patients with congenital disturbances as hyperplasia or hypoplasia of the joint.

g. Patients with osteo arthritic changes in TMJ bony components.

h. Patients with loss of normal TMJ disc architecture (over thinning or perforations). 
i. Patients contraindicated to MRI (as patients with pacemakers, cerebral aneurismal clips, artificial cardiac valves, metal implants, hearing aids, and claustrophobic patients).

\section{Preoperative Preparations:}

- Medical, dental histories, and history of chief complaint were taken.

- All selected patients were informed about the procedure,precautions,follow up appointments, possible complications. Also, they signed informed consent.

\section{Preoperative clinical evaluation:}

- Pain scoring was carried out through visual analogue scale (VAS) with 0 score for no pain and10 score for worst pain experienced.

- Using a digital caliper,maximal unassisted mouth opening, Lateral, and protrusive movements were measured in millimeters.

- Joint sounds as clicking or crepitation were evaluated preoperatively by palpation. The clicking was reported for each joint if present.

- All these measurements records were considered as a baseline to be used in comparison with post-operative records.

\section{Preoperative MRI Examination}

All patients were evaluated using MRI (open and closed) to diagnose internal derangement. The MRI was carried out in a private radiology center usinga 1.5T MR scanner (Gyroscan Intera Master; Philips Healthcare, Eindhoven, The Netherlands) and a dedicated, circular polarized transmit and receive TMJ coil. (Figure 9) The MRI protocol included bilateral sagittal oblique proton density images of the right and left sides in both the closed mouth (maximum intercuspation) and maximum mouth opening positions. The examination also included bilateral coronal proton density images of the right and left sides at the closed mouth position.

\section{Drugs used with operative procedures}

1. Simvastatin: (calbiochem): $1 \mathrm{mg} / \mathrm{ml}$ Ampoule, Manufactured by EMD Millipore Crop., Billerica, MA USA. The injected dose was $(1 \mathrm{mg} / \mathrm{ml})$.

2. Sodium Hyaluronate: (Curavisc): $20 \mathrm{mg} / 2 \mathrm{ml}$ Syringe, Manufactured by IDT Biologika GmbH Company, Germany. The injected dose was $(1 \mathrm{mg} / \mathrm{ml})$.

\section{Operative procedures :}

\section{Arthrocentesis procedure:}

- All patients carried out arthrocentesis procedure before injection material according to Nitzan ${ }^{(7)}$ technique by drawing the canthal-tragus line and a point $10 \mathrm{~mm}$ in front of the tragus and 2 $\mathrm{mm}$ below the canthal-tragus line was marked to serve as the point of insertion of the first needle used as an inflow needle (red dot). Another point $20 \mathrm{~mm}$ in front of the tragus and $8 \mathrm{~mm}$ below the canthal-tragus line (i.e. $10 \mathrm{~mm}$ anterior to the former one) was marked to serve as the point of insertion of the second needle used as an outflow needle (green dot) (Figure 1).

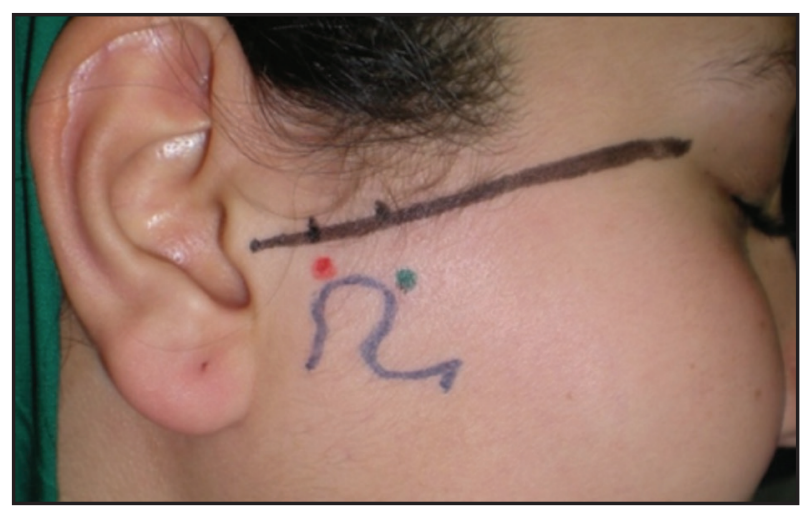

Fig. (1): Target areas marked with a washable felt-tip pen 
- Local anesthesia of auriculotemporal nerve was administrated using 0.3 to $0.5 \mathrm{ml}$ of an anesthetic solution 2\% Mepivacaine with 1/200000 Levonordefrin.

- A 20-gauge needle was inserted at the marked first point and another one in the second marked point (Figure 2a).

- The joint was lavaged with $100-300 \mathrm{ml}$ ringer lactate solution injected into the upper joint compartment through inflow needle; the outflow needle was periodically occludedto create hydraulic pressure within the joint space. The patients were asked to open and close their mouth during the procedure to help outflow of the injected ringer lactate.

\section{Injection of drugs:}

Group I: (Study group)

After arthrocentesis had been performed for the affected joints, One $\mathrm{ml}$ of simvastatin containing $(5 \mathrm{mg})$ was injected intra-articularly. Patients were then asked to open their mouth and a needle was inserted at two target areas,the first target area was the posterior joint space and the second target is the anterior disc attachment. The standard program was to repeat the injections two times, at one month interval( Figure2b).

\section{Group II: (Control Group)}

After arthrocentesis had been performed for the affected joints, one $\mathrm{mL}$ of commercially available Sodium Hyaluronate containing $(10 \mathrm{mg})$ was injected intra-articularly. $2 \mathrm{ml}$ readymade syringes were used for its injection into the same previous points as in group I.The standard program is to repeat the injections two times, at one month interval (Figure2 c).

\section{Postoperative care and followup:}

All patients were asked to follow the following instructions:

- Place cold application in the form of ice bag extra orally for ten minutes every half an hour for the first 24 postoperative hours.

- Place hot fomentation extra-orally for ten minutes every half an hour for 1 week postoperatively.

- Maintain soft diet and avoid eating hard food, gum chewing for 1 week post operatively.

- Gradually transform to a normal diet within the first postoperative week.

- Initiate physiotherapy exercises immediately postoperative.

- Drugs prescribed: Analgesic (brufen $600 \mathrm{mg}$ ) every $12 \mathrm{~h}$ for 3 days.

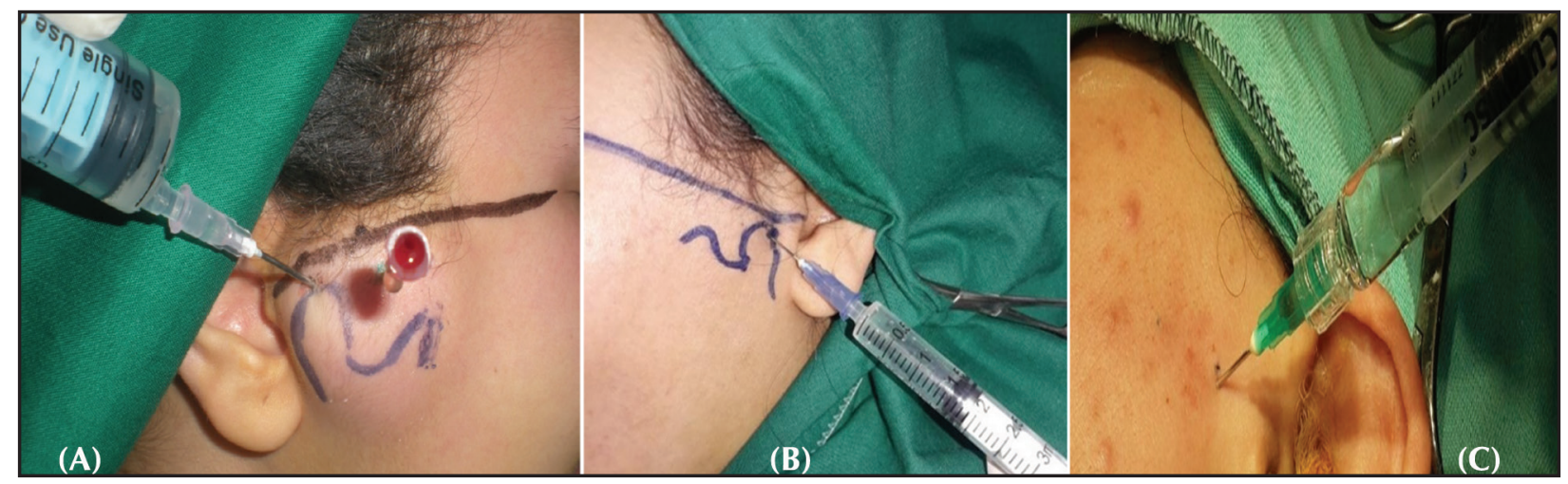

Fig. (2): A. Needles inserted at marked points. B. Injection of Simvastatin. C. Sodium hyaluronate injection 


\section{Clinical followup:}

The postoperative clinical evaluation carried out at 24 hours postoperatively, 1 week, 1 month and 6 month after the procedure to assess pain level, maximal mouth opening, lateral excursion movements, protrusive movements, joint sound.

\section{MRI followup:}

MRI was taken 6 months postoperatively to assess disc displacement,disc position, disc shape, and retrodiscal tissues.

\section{Statistical analysis:}

Numerical datawere collected and presented as mean and standard deviation values. Two-way ANOVA (analysis of variance) was used to evaluate the effect of time on patients in both groups. The significance level was set at $\mathrm{P} \leq 0.05$.

Statistical analysis was performed with SPSS17.0 ${ }^{\circledR}$ (Statistical Package for Scientific Studies) for Windows. The significance of the difference between both groups was evaluated using unpairedt-test for independent samples.

\section{RESULTS}

The study included 30 patients (19 female and 11 male ) with ages ranged from 19 to 34 years. All patients tolerated injection procedures without any complications.

\section{Pain score:}

There was no significant difference between group I and II at preoperatively and 24 hours postoperative. Regarding pain scores at all post-operative observation times; there was a significant difference between both groups ( $\mathrm{p}<0.0001)$, withhigher pain values observed in group II at all time intervals.(Table1). Preoperative measurements of pain score in group I revealed $8.15 \pm 0.94$ and in group II
$8.05 \pm 0.82$. There was a significant decrease in pain score after 24 hours as it was in group I $6.90 \pm 1.55$ (P-value< 0.0001 ) and in group II $6.80 \pm 1.19$. There was also a decrease in pain score in group I after 1 week to be $5.20 \pm 1.10$ and in group II $7.60 \pm 0.94$, after 1 month pain score in group I was decreased to be $4.20 \pm 0.69$ and in group II $5.75 \pm 1.16$ and after 6 months it was in group I $1.05 \pm 0.68$ and in group II 2.75 \pm 0.71 (Table1).

\section{Mouth opening:}

There was no significant difference between group I and II preoperatively and 24 hours postoperative. However, in postoperative observation times 1 week, 1 month, and 6 months; there was a significant difference between both groups, with higher values observed in group I at all time intervals $(p<0.0001)$. Preoperative measurements of mouth opening in group I revealed 19.9 \pm 5.38 and in group II 17.20 \pm 3.51 . There was a significant decrease in mouth opening after 24 hours as it was in group I 17.4 \pm 4.37 (P-value $<0.0001)$ and in group II $16.70 \pm 2.34$. There was also decrease in mouth opening in group I after 1 week to be $24.00 \pm 5.40$ and in group II $15.95 \pm 3.15$, after 1 month mouth opening in group I was decreased to be $31.6 \pm 2.60$ and in group II $22.45 \pm 3.37$ and after 6 months it was in group I $33.75 \pm 1.11$ and in group II $30.40 \pm 2.06$ (Table 1).

\section{Lateral excursion:}

Preoperative measurements of lateral excursion in group I revealed $14.15 \pm 3.28$ and in group II $13.80 \pm 1.84$. There was a decrease in lateral excursion after 24 hours as it was in group I 13.6 \pm 2.46 (P-value $<0.0001$ ) and in group II $12.10 \pm 3.34$ with no statistically significant difference between both groups in the mean values. There was an increase in the lateral excursion in group I after 1 week to be $15.50 \pm 2.60$ and in group II $15.30 \pm 2.54$, after 1 month lateral excursion in group I increased to be $21.40 \pm 2.89$ and in group II $20.30 \pm 2.99$ and 
after 6 months it was in group I $23.25 \pm 1.86$ and in group II $22.10 \pm 1.52$ with no statistically significant difference between both groups in the mean values (Table 1).

\section{Protrusive movement:}

Preoperative measurements of protrusive movement in group I revealed $11.80 \pm 3.16$ and in group II 11.25 \pm 1.97 . After 24 hours it was in group I $10.35 \pm$ 2.21 (P-value $<0.0001$ ) and in group II 10.45 \pm 2.04 . After 1 week, protrusive movement values in group I was $13.80 \pm 1.88$ and in group II $13.73 \pm 2.65$, after 1 month protrusive movement in group I increased to be $17.85 \pm 2.23$ and in group II $15.65 \pm 1.93$ with a statistically significant difference between both groups. Similarly, after 6 months values were in group I $21.25 \pm 1.55$ and in group II $16.75 \pm 2.00$ (Table 1).

\section{Joint sounds:}

Regarding joint soundsscores at all post-operative observation times 1 week, 1 month, and 6 months; there was a significant difference between both groups $(\mathrm{p}<0.0001)$, with higher joint sounds values recorded in group II at all time intervals. Preoperative measurements of joint sounds score in group I revealed $8.15 \pm 0.94$ and in group II $8.05 \pm 0.82$. There was a significant decrease in joint sounds score after 24 hours as it was in group I $6.90 \pm 1.55$ (P-value $<0.0001)$ and in group II 6.80 \pm 1.19 . There was also decrease in joint sound score in group I after 1 week to be $5.20 \pm 1.10$ and in group II $7.60 \pm$ 0.94 , after 1 month joint sounds score in group I was decreased to be $4.20 \pm 0.69$ and in group II $5.75 \pm 1.16$ and after 6 months it was in group I $1.05 \pm 0.86$ and in group II $2.75 \pm 0.71$ (Table1).

Table(1): Comparison between clinical parameters of both groups

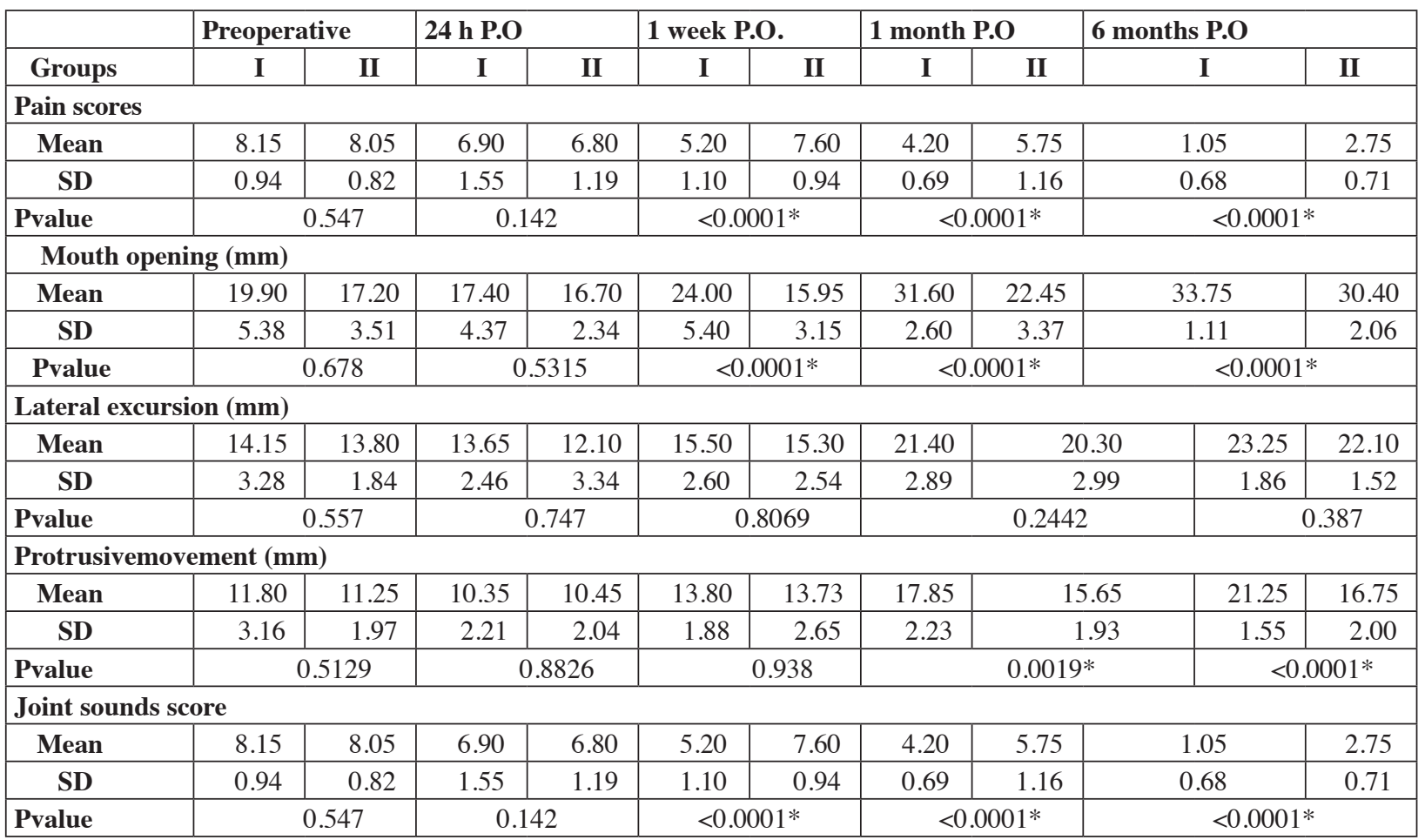

P.O. = postoperatively,

* Statistically significant at $p<0.05$ 


\section{MRI results:}

Analysis of MRI finding postoperatively showed no significant difference between both groups regarding all examined MR criteria except in normal disc position and biconcave disc shape where a significant difference was found. In normal disc position, group I was 14 joints (46.7\%), group II was 12 joints (40\%).While in biconcave disc shape group I was 20 joints (66.7\%), group II was 12 joints (40\%) (figure 3\&4) ( Table2).

Table(2): Values of postoperative MRI Findings in both groups

\begin{tabular}{|c|c|c|c|c|c|c|}
\hline \multirow{2}{*}{\multicolumn{2}{|c|}{ MRI findings of the disc No. Post }} & \multicolumn{2}{|l|}{ Group I } & \multicolumn{2}{|r|}{ Group II } & \multirow{3}{*}{$\begin{array}{l}\text { p-value } \\
0.07\end{array}$} \\
\hline & & \multirow{2}{*}{\begin{tabular}{|l|}
$\%$ post \\
16 \\
\end{tabular}} & \multirow{2}{*}{$\begin{array}{l}\text { No. Post } \\
53.3 \\
\end{array}$} & $\%$ post & & \\
\hline Dico lialo & Anterior & & & 12 & 40 & \\
\hline Dise aispiactintit & Anteromedial & 8 & 26.7 & 6 & 20 & 0.053 \\
\hline \multirow{3}{*}{ Disc position } & Normal & 14 & 46.7 & 12 & 40 & $<0.0001 *$ \\
\hline & Intermediate zone & 4 & 13.3 & 4 & 13.3333333 & 0.05 \\
\hline & Posterior band & 2 & 6.7 & 2 & 6.66666667 & 0.05 \\
\hline \multirow{2}{*}{ Disc shape } & Biconcave & 20 & 66.7 & 12 & 40 & $<0.0001 *$ \\
\hline & Hemiconvex & 4 & 13.3 & 6 & 20 & 0.067 \\
\hline Retrodiscal tissue & Normal & 30 & 100.0 & 30 & 100 & - \\
\hline
\end{tabular}

* Statistically significant at $p<0.05$

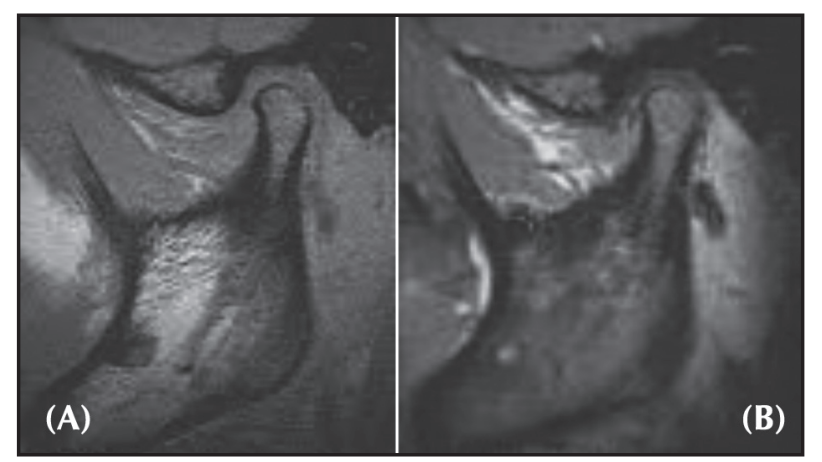

Fig. (3): Sagittal MRI in case of group I (open-mouth position) shows that (A) Articular disk displaced anteriorly to condyle in preoperative MRI (B) postoperative MRI shows the intermediate zone of the disc (solid arrow) is located between the condyle and the articular

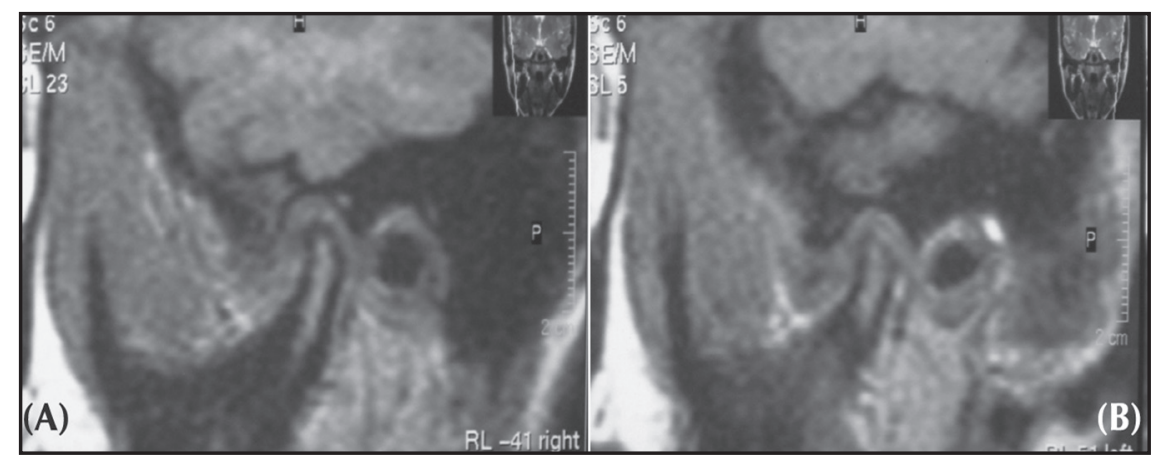

Fig. (4): Sagittal MRI in case of group II (open-mouth position) shows that (A) Articular disk displaced anteriorly to condyle in preoperative MRI (B) postoperative MRI shows the intermediate zone of the disc is located between the condyle and the articular eminence. 


\section{DISCUSSION}

TMJ disorders are common in the population and they may strongly affect the quality of life,being male or female is one of the most important predictors of an individual. The treatment can range from a conservative intraarticular injection of medication that is preferred firstly than surgical intervention $^{(10,11)}$

No clinical studies were done to evaluate the effect of intra-articular injection of simvastatin in internal derangement in humans. So the present study aimed to assess and compare intra-articular injection of simvastatin versus sodium hyaluronate after arthrocentesis for management of internal derangement of TMJ.

In the present study, the patients' ages ranged from 20 to 34 years, this may be explained by those patients who have more responsibility in family, society, labor,and bearing many life stresses. Therefore, most studies revealed this group to show a higher incidence of TMJ complaints. The selected age group was in accordance with Choi et al, ${ }^{(12)}$ who found that patients between second to third decade of life had more symptoms of TMJ dysfunction and Mohajerani et al, ${ }^{(13)}$ who found that the age group from 27-35 was the most common group to have TMDs signs.

In 2009, Zhang et al, ${ }^{(14)}$ had concluded that local injection of simvastatin-loaded on a gel vehicle of polyethylene glycol-poly lactic acid at a concentration of $(5 \mathrm{mg} / \mathrm{ml})$ had a role in relieving pain and promotion of autogenous chondrogenic intervertebral discrepair and retard disc degeneration, which provides an alternative modality for biological disc repair in a less expensive and easily applied method. From this point, simvastatin was strongly suggested to be injected intraarticulary for the repair of internal derangement in humans.
Regarding the recommended dose of simvastatin injection,Than et al, ${ }^{(15)}$ in 2014 had demonstrated greater benefit of $5 \mathrm{mg} / \mathrm{ml}$ simvastatin in hydrogel than higher doses of $10 \mathrm{mg} / \mathrm{ml}$ or $15 \mathrm{mg} / \mathrm{ml}$. They had claimed that higher doses were toxic to the disc secondary to altered cell membrane adipose metabolism, resulting incell death. This was matched with injection dose in the current study where $1 \mathrm{mg} / \mathrm{ml}$ simvastatin was injected intraarticulary in the superior joint space twice per month and getting a gradual decrease in pain score starting from 24 hours postinjection that continued up to six months.

In the present study, both groups I \&II had better comparable results regarding the decrease in pain intensity. So, pain was found to be the most common clinical finding affecting patients with internal derangement. This was in accordance with Cooper and Kleinberg ${ }^{(16)}$. However, this was in contrary to Gesch $^{(17)}$ who found that the most common clinical finding was deviation of the mandible followed by limitation of opening followed by pain. Similarly, Marklund and Wanman ${ }^{(18)}$ reported that the most common clinical finding was joint sound followed by pain. Mohajerani et al, ${ }^{(13)}$ also stated that the most common clinical finding was joint sound followed by limitation of mouth opening followed by pain.

These results were also in agreement with Yakan et al. ${ }^{(19)}$ study that assessed the efficacy of arthrocentesis and hyaluronic acid injections in the treatment of TMJ osteoarthritis in 20 patients who underwent the first session consisted of arthrocentesis plus injection of hyaluronic acid, followed by four sessions of hyaluronic acid injection only).

Additionally, both lateral excursions and protrusive movements had shown a significant variable difference in group I than group II. This can be explained by the fact that harmony of disc condyle relationship was achieved dramatically 
after injection of simvastatin that denotes improvement in both lateral excursions and protrusive movements. This was in accordance with GilC. et al. ${ }^{\left({ }^{20)}\right.}$ who claimed delayed improvement in both lateral excursion and protrusive movement after hyaluronic acid injection.

Regarding maximum mouth opening,the present study showed no significant difference in both groups through different postoperative intervals. However, there may be a slight improvement in mouth opening in group I than group II after the first week from injection that was attributed to the persistence of pain after washing effect of HA from the joint that affects the range of motion. These results were inaccordance with studies performed by; Zhang et al, ${ }^{(14)}$ and Than et al, ${ }^{(15)}$ in which they proposed that simvastatin in hydrogel $5 \mathrm{mg} / \mathrm{ml}$ injection can improve the regeneration capacity of the degenerated intervertebral discs.

Joint sounds were reported before and after treatment by injection of both simvastatin and HA. It was observed that joint sounds followed the same curve of pain intensity which means that whenever pain was remarkable joint sound was markedly noticed. After the pain was relieved the joint sounds almost disappeared which reflects the correlation between pain and joint sounds. This was in agreement with Marklund and Wanman ${ }^{(18)}$.

Six months postoperatively MRI examination revealed that patients of group I showed normal orientation of disc position and shape following simvastatin injection while in group II there was abnormal orientation of disc position and shape in relation to articular eminence and glenoid fossa. Regarding MR imaging of both groups, it was found that there was improvement in several signs of internal derangement. This was in accordance with Park et al, ${ }^{(21)}$ who concluded similar results.

\section{CONCLUSION}

Finally, it has been concluded that arthrocentesis followed by simvastatin intra-articular injection was superior to the combination of arthrocentesis and sodium hyaluronate intra-articular injection for management of TMJ internal derangements symptoms. Where, simvastatin intra-articular injection proved to be able to achieve relief of pain in patients with TMJ internal derangement,allowing patients to perform their mandibular functions freely without pain.

\section{REFERENCES}

1. Hase M. Adhesions in the Temporomandibular Joint: Formation and Significance. Aust Dent J 2002;47(2):163169.1

2. Byun ES, Ahn SJ, Kim TW. Relationship between Internal Derangement of the Temporomandibular Joint and Dentofacial Morphology in Women with Anterior Open Bite. Am J Orthod Dentofacial Ortho P 2005;128:87-95.

3. Shahin A K A,ELSharrawy E A, AbdElHamid AS, Hamed T A.The Role of Relaxin Hormone in Internal Derangement Of temporomandibular Joint. ZUMJ 2013;19(5):317-22.

4. Manfredini D, Rancitelli D, Nardini L G. Arthrocentesis in Temporomandibular Joint Inflammatory Degenerative Disease: A randomized and Controlled Trial. J.of Cranio Func 2011;3(1):41-46.

5. Vasconcelos BC, Nogueira RV, and Rocha NS. Temporomandibular Joint Arthrocententesis: Evaluation of Results and Review of the Literature. Rev Bras Otorrinolaringol 2006;72(4):634-8.

6. Draeger HT, Twining JM, Johnson CR, KettwichS C, KettwichL G, Bankhurst AD. A Randomized Controlled Trial Of The Reciprocating Syringe In Arthrocentesis. AnnRheumDis 2006;10(1136):1-4.

7. Guarda-Nardini L, Stifano M, Brombin C, Salmaso L, Manfredini D. AOne-Year Case Series of Arthrocentesis with Hyaluronic Acid Injections for Temporomandibular Joint Osteoarthritis. Oral Surg Oral Med Oral Pathol Oral Radiol Endod 2007;103: 14-22. 
8. H Quasnichka, E Budd, J Tarlton, S Roberts, C Jackson, B Caterson, A Mobasheri. The effect of simvastatin on novel exploratory biomarkers of cartilage degradation. Osteoarthritis and cartilage 2018; Volume 26, Supplement 1, Page S19226-560 - 5474.

9. Nitzan DW, Dolwick MF, Martinez GA.Temporomandibular Joint Arthrocentesis: A Simplified Treatment for Severe, Limited Mouth Opening. J Oral Maxillofac Surg 1991; 49:1163-7.

10. Kohler A A.On Temporomandibular Disorders Time Trends, Associated Factors, Treatment Need,and Treatment Outcome. School of Health Sciences,Swed. Dent. J 2012;38:227.

11. Solberg W K, WooM W, Houston J B. Prevalence of Mandibular Dysfunction in Young Adults. Am Dent Assoc J 1979;98(1):25-34.

12. Choi Y S, Asaumi J I, Hisatomi M, Unetsubo T, Yanagi Y, Matsuzaki H, Konouchi H, Hwang EH, and Lee SR. Analysis of magnetic resonance images of disk positions and deformities in 1,265 patients with TMD. Open Dent J.2009; 3:1-20.

13. Mohajerani S, Ghorbani Z, Gholami L, Tavakolizadeh S, and Ebrahimzadeh Z. Prevalence of TMDs among patients referred to Shaheid Beheshti dental school, Iran. Dent J Ham 2011;2(1):1-9.

14. Zhang H, Wang L, Park J B, Park P, Yang VC, Hollister SJ, et al. Intradiscal injection of simvastatin retards progression of intervertebral disc degeneration induced by stab injury. Arthritis Res Ther 2009;11(6):R172.
15. Than KD, Rahman SU, Wang L, Khan A, Kyere KA, Than TT, etal. Intradiscal injection of simvastatin results in radiologic, histologic, and genetic evidence of disc regeneration in a rat model of degenerative disc disease. Spine J. 20171; 14(6):1017-28.

16. Cooper BC, Kleinberg I. Examination of a large patient population for the presence of symptoms and signs of TMDs. Cranio 2007;25(2):114-26.

17. Gesch D, Bernhardt O, Mack F, John U, Kocher T, and Alte D.Dental occlusion and subjective TMJ symptoms in men and women. Results of the Study of Health in Pomerania (SHIP). Schweiz Monatsschr Zahnmed 2004;114(6):573-80. 122.

18. Marklund S, Wänman A. Incidence and prevalence of TMJ pain and dysfunction. A one-year prospective study of university students. Acta Odontol. Scand. 2007;65(2):119-27.

19. Yakan A,Toameh M. Treatment of Temporomandibular Joint Osteoarthritis by Using Arthrocentesis and Hyaluronic Acid Injections: Modified Protocol. J Dent Health Oral Disorder 2014;1(5):1-6.

20. Gil C, Santos KC, Dutra ME, Kodaira SK, Oliveira JX. MRI Analysis of the Relationship between Bone Changes in The Temporomandibular Joint and Articular Disc Positionin Symptomatic Patients. Br. Inst. of Dentomaxillofac Rad. 2012;41:367-372.

21. Park JW, Song HH, Roh HS, Kim YK, and Lee JY. Correlation between clinical diagnosis based on RDC/ TMD and MRI findings of TMJ internal derangement. Int J Oral Maxillofac Surg 2012;41(1):103-8. 